\title{
Images of the month 1: An unusual case of breathlessness - coronary artery fistula draining into the left ventricle
}

Authors: Nida Ahmed, ${ }^{A}$ Sabahat Ahmed, ${ }^{B}$ Riyaz Patel ${ }^{C}$ and Mohammed Khanji ${ }^{\mathrm{D}}$

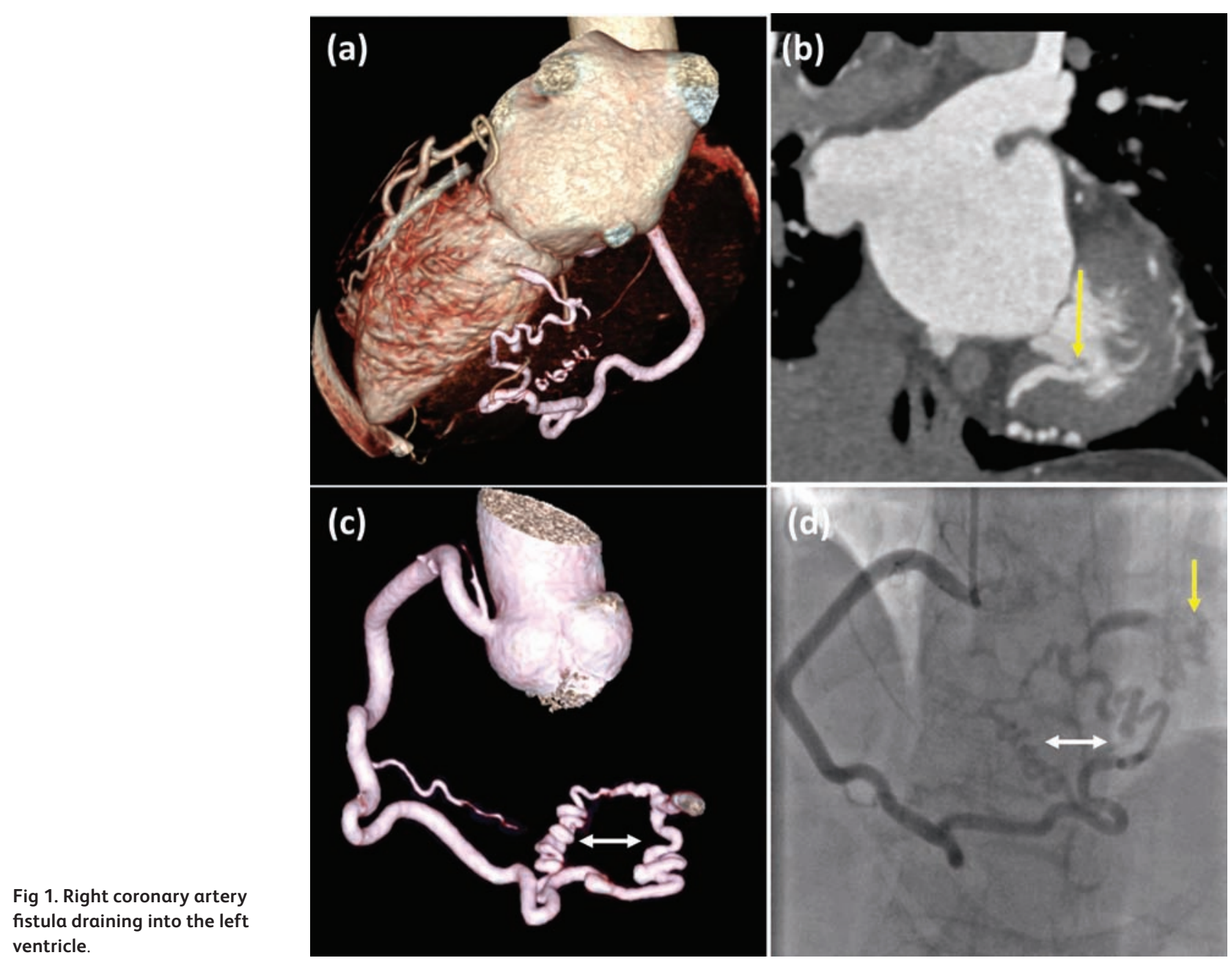

\section{Case}

A 68-year-old woman presented to cardiology clinic with chest pain and longstanding, yet stable, breathlessness

Authors: ${ }^{\text {A }}$ core medical trainee year 2, Barts Health NHS Trust, London, UK; ${ }^{B}$ medical student, GKT School of Medicine, London, UK; C Consultant cardiologist, Barts Health NHS Trust, London, UK; ${ }^{D}$ consultant cardiologist, Barts Health NHS Trust, London, UK limiting her to 5 minutes of moderate exertion. Her mobility was also limited by pain from lumbosacral spondylosis, knee osteoarthritis and osteoporosis. She had no cough, orthopnoea or peripheral oedema. Cardiovascular risk factors included hypercholesterolaemia and hypertension. She had iron-deficiency anaemia due to gastritis.

Cardiovascular examination was unremarkable. Blood pressure was normal $(137 / 75 \mathrm{mmHg})$ and body mass index was $26 \mathrm{~kg} / \mathrm{m}^{2}$. Heart sounds were normal with no murmurs and no signs of fluid overload. Blood test showed iron-deficiency anaemia (normal 
vitamin B12 and folate levels), which later improved (107 g/L to $121 \mathrm{~g} / \mathrm{L}$ ) with iron supplements.

Electrocardiogram was sinus rhythm with no evidence of ischemia. Computed tomography with coronary angiography (Figs 1b and d, yellow arrow), including 3-D reconstruction (Figs 1a and C), showed an arteriovenous fistula draining into the left ventricle (LV) with unobstructed coronary arteries. Subsequent invasive coronary angiography confirmed the tortuous vessels (Fig 1d, white arrow) flow into the LV. Her echocardiogram demonstrated normal biventricular size and function and no obvious flow from the fistula into the LV. Though limited by her breathlessness, she did not want to consider closure and was managed conservatively.

\section{Discussion}

Coronary arteriovenous fistula (CAVF) is an uncommon finding, occurring in $0.002 \%$ of the population, often found incidentally at echocardiography or coronary angiography. Associated complications include endocarditis, dissection and high output cardiac failure. When acquired, it can be secondary to cardiac intervention procedures. CAVFs can be managed either through careful observation, percutaneous fistula closure or surgical ligation. Repair is indicated where there is demonstrable ischemia or shunt-related symptoms eg breathlessness. ${ }^{1}$ It is important to monitor closely in those not undergoing intervention; demonstrated in one case where the patient was lost to followup and subsequently demonstrated a tripling in the size of the coronary artery in the 30 years after the identification of the lesion. ${ }^{2}$

\section{References}

1 Rahman F, Cookman B, Tumuluri R, Ammar KA. Massively enlarged right coronary artery with fistula to the coronary sinus: To close or not to close? J Am Coll Cardiol 2016;67(13 Suppl):1201.

2 Chaumont M, Unger P, Debbas N, Vandenbossche J. Unoperated giant coronary arteriovenous fistula: A thirty-year follow-up. Eur Heart ] 2016:38:217.

Address for correspondence: Dr Nida Ahmed, St Bartholomew's Hospital, West Smithfield, London EC1A 7BE, UK. Email: nida_3683@hotmail.com

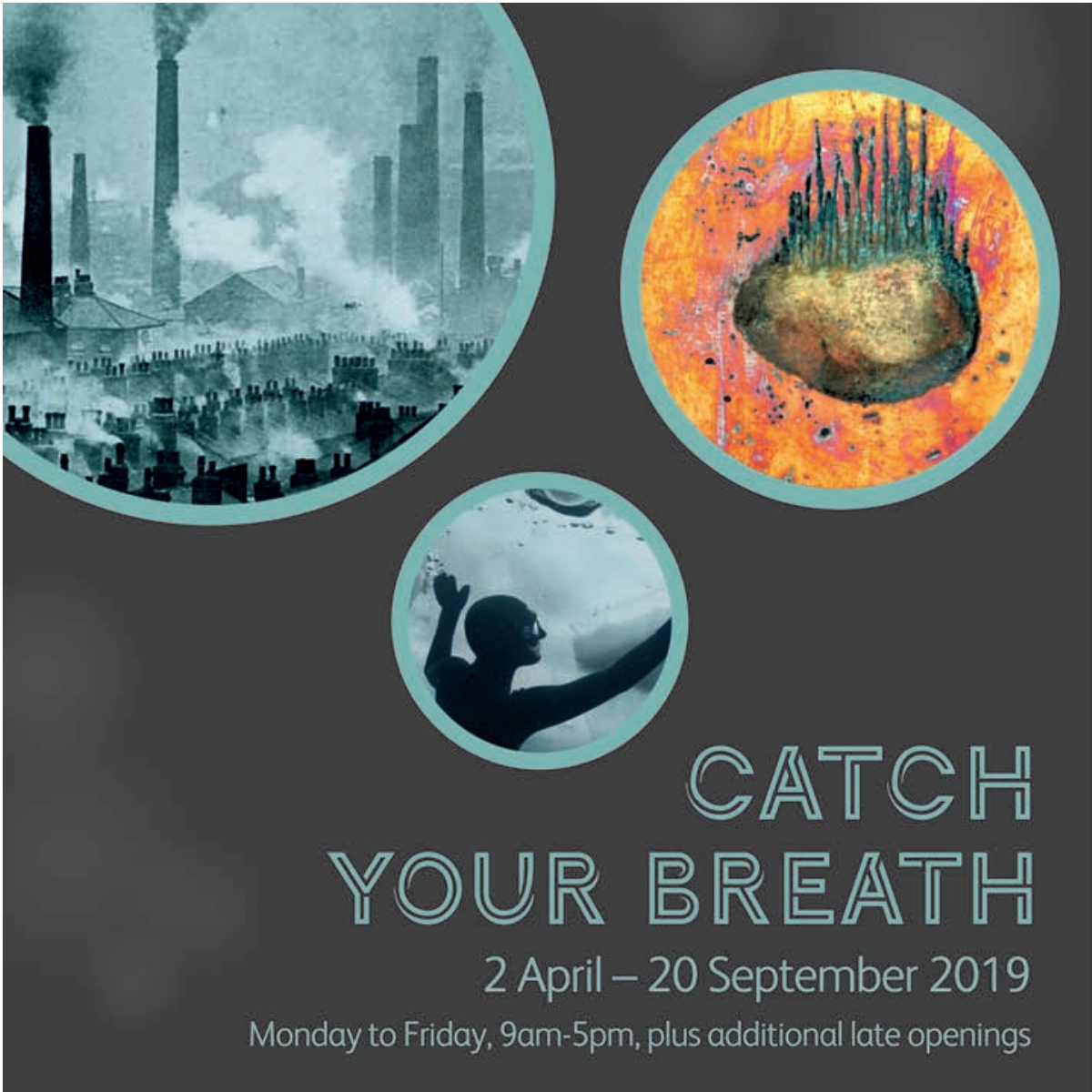

\section{TEMPORARY EXHIBITION}

Royal College of Physicians 11 St Andrews Place Regent's Park London NW1 4LE

\#CatchYourBreath @RCPmuseum @LifeofBreath history.rcplondon.ac.uk/events catchyourbreath.org

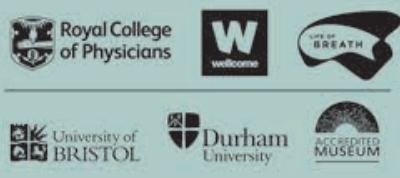

